\title{
Scattering formalism in the Casimir interaction of gratings and Chern-Simons layers
}

\author{
Valery N. Marachevsky ${ }^{1, *}$ \\ ${ }^{1}$ Department of Theoretical Physics, Saint Petersburg State University, Ulianovskaya 1, \\ Petrodvorets, 198504 St. Petersburg, Russia
}

\begin{abstract}
We describe scattering formalism to evaluate the Casimir energy of two gratings separated by a vacuum slit and the Casimir energy of two ChernSimons layers in vacuum.
\end{abstract}

\section{Introduction}

The Casimir effect is a quantum fluctuation effect in the presence of boundaries [1] - [7].

In $[8,9]$ it was shown that the Casimir force of two flat Chern-Simons layers in vacuum can be both attractive and repulsive depending on values of constants in Chern-Simons actions. Repulsive Casimir forces appear in interaction of two Chern-Simons layers due to mixing of TE and TM modes during diffraction process. The Casimir-Polder potential of a neutral anisotropic atom in the presence of a flat Chern-Simons layer was found in [10], charge-parity violating effects in Casimir-Polder potentials were studied in [11].

Systems with Chern-Simons terms on their surfaces have unique properties since a stable minimum of the Casimir energy naturally arises at nanoscales [12]; this feature should allow an effective experimental study of interactions beyond electromagnetism via the Casimir force measurements in future.

The theory of two gratings with $1 \mathrm{~d}$ spatial periodicity was developed in papers $[13,14]$, the Casimir free energy was expressed in terms of Rayleigh coefficients [15]. The theory was found to be in agreement with experimental measurements of the lateral Casimir force between two sinusoidal 1d Au gratings with different amplitudes [16].

In the present paper we describe scattering formalism which found itself effective in studies of both systems. The approaches to evaluate the Casimir energy of gratings and ChernSimons layers are similar from the mathematical point of view. This similarity may be essential for better understanding of repulsive and attractive forces in the Casimir effect.

An outline of the paper is the following. In Sec. 2 we introduce a general formalism for evaluation of Rayleigh coefficients for 1d gratings and express the Casimir free energy of two gratings with coinciding periods in terms of Rayleigh coefficients. In Sec. 3 we solve a diffraction problem for a plane Chern-Simons layer in vacuum. In Sec. 4 the Casimir energy of two plane Chern-Simons layers is derived.

We use $\hbar=c=k_{B}=1$ throughout the paper.

\footnotetext{
*e-mail: maraval@mail.ru
} 


\section{Casimir energy of $1 \mathrm{~d}$ gratings}

Consider two 1d gratings which are periodic in a direction $x$ with a period $d$ and separated by a vacuum slit $L$ which is a minimum distance between the gratings. Materials of the gratings are defined by frequency dependent permittivities, the condition $\mu=1$ for magnetic permeability is assumed in the whole space. Periods of the gratings in a selected spatial direction coincide. To obtain reflection matrices for systems with periodic surface profiles or spatially periodic material properties it is convenient to consider Rayleigh decompositions [15]. The formalism is applicable to arbitrary surface profiles of 1d gratings.

Consider diffraction of an electromagnetic plane wave on a single grating. For $1 \mathrm{~d}$ periodic geometries the solutions of Maxwell equations can be decomposed in Fourier series on a variable $x$. The material of the grating is described by a frequency dependent dielectric permittivity $\varepsilon(\omega)$. Suppose $h$ is the height of the grating's corrugation region $z \in[-h, 0]$ (periodic in direction $x$ and translationally invariant in direction $y$ ) while for $z<-h$ grating's dielectric permittivity is equal $\varepsilon(\omega)$ at all spatial points. One can write exact solutions of Maxwell equations for $z>0(\varepsilon=1$ for $z>0)$ in terms of Rayleigh reflection coefficients $R_{n p}^{(e)}, R_{n p}^{(m)}\left(e^{-i \omega t+i k_{y} y}\right.$ factors are omitted $)$ :

$$
\begin{aligned}
& E_{y}(x, z)=I_{p}^{(e)} \exp \left(i \alpha_{p} x-i \beta_{p}^{(1)} z\right)+\sum_{n=-\infty}^{+\infty} R_{n p}^{(e)} \exp \left(i \alpha_{n} x+i \beta_{n}^{(1)} z\right), \\
& H_{y}(x, z)=I_{p}^{(m)} \exp \left(i \alpha_{p} x-i \beta_{p}^{(1)} z\right)+\sum_{n=-\infty}^{+\infty} R_{n p}^{(m)} \exp \left(i \alpha_{n} x+i \beta_{n}^{(1)} z\right) .
\end{aligned}
$$

Here $\alpha_{p}=k_{x}+2 \pi p / d$ and $\beta_{p}^{(1) 2}=\omega^{2}-k_{y}^{2}-\alpha_{p}^{2}$, $d$ is a period of gratings, $k_{x} \in[-\pi / d, \pi / d]$, $p \in Z$.

For $z<-h$ Rayleigh decompositions for electromagnetic field can be written in terms of transmission coefficients $T_{n p}^{(e)}, T_{n p}^{(m)}$.

Consider the vector of fourier components $E_{x}, E_{y}, H_{x}, H_{y}$. In numerical calculations it is sufficient to consider fourier components with integer numbers $n$ possessing values $n \in$ $[-N, N]$, values of $N$ are determined by the accuracy of calculations. Let's denote by $\left[E_{x}\right]$ the vector with $(2 N+1)$ fourier components of the field $E_{x}$, vectors for other components of the electromagnetic field are denoted in analogy.

In the range of values $-h<z<0$ Maxwell equations can be rewritten in the form of first order differential equations [17]:

$$
\partial_{z}\left(\begin{array}{l}
{\left[E_{x}\right]} \\
{\left[E_{y}\right]} \\
{\left[H_{x}\right]} \\
{\left[H_{y}\right]}
\end{array}\right)=M\left(\begin{array}{l}
{\left[E_{x}\right]} \\
{\left[E_{y}\right]} \\
{\left[H_{x}\right]} \\
{\left[H_{y}\right]}
\end{array}\right),
$$

$M$ is a square matrix depending on $i \omega, k_{x}, k_{y}$ and surface profile in the range $-h<z<$ 0 . At $z=-h, z=0$ the continuity conditions are imposed on all Fourier components of longitudinal fields $E_{x}, E_{y}, H_{x}$ and $H_{y}$. As a result, the system of linear equations on the Rayleigh coefficients $R^{(e)}, R^{(m)}, T^{(e)}, T^{(m)}$ can be obtained.

The reflection matrix from the lower grating is constructed as follows:

$$
R_{1 \text { down }}(\omega)=\left(\begin{array}{ll}
R_{n_{1} l_{1}}^{(e)}\left(I_{p}^{(e)}=\delta_{p l_{1}}, I_{p}^{(m)}=0\right) & R_{n_{2} l_{2}}^{(e)}\left(I_{p}^{(e)}=0, I_{p}^{(m)}=\delta_{p l_{2}}\right) \\
R_{n_{3} l_{3}}^{(m)}\left(I_{p}^{(e)}=\delta_{p l_{3}}, I_{p}^{(m)}=0\right) & R_{n_{4} l_{4}}^{(m)}\left(I_{p}^{(e)}=0, I_{p}^{(m)}=\delta_{p l_{4}}\right)
\end{array}\right) .
$$

Note that Rayleigh decomposition is exact outside gratings, the Rayleigh coefficients are determined from exact solution of Maxwell equations. 
For evaluation of the Casimir free energy we need matrices of reflection coefficients from the lower and upper gratings. Suppose the second (upper) grating is located at $z>L$, the distance $L$ is then a minimum distance between the gratings.

By making use of argument principle

$$
\frac{1}{2 \pi i} \oint \phi(\omega) \frac{d}{d \omega} \ln f(\omega) d \omega=\sum \phi\left(\omega_{0}\right)-\sum \phi\left(\omega_{\infty}\right)
$$

with

$$
\phi(\omega)=\omega / 2
$$

and

$$
f(\omega)=\operatorname{det}\left(I-R_{1 \text { down }}\left(\omega, k_{x}, k_{y}\right) R_{2 u p}\left(\omega, k_{x}, k_{y}\right)\right)
$$

one derives the Casimir energy of two gratings in the form [13]:

$$
E=\frac{1}{(2 \pi)^{3}} \int_{0}^{+\infty} d \omega \int_{-\infty}^{+\infty} d k_{y} \int_{-\pi / d}^{\pi / d} d k_{x} \quad \ln \operatorname{det}\left(I-R_{1 d o w n}\left(i \omega, k_{x}, k_{y}\right) R_{2 u p}\left(i \omega, k_{x}, k_{y}\right)\right)
$$

Here

$$
R_{2 u p}\left(i \omega, k_{x}, k_{y}\right)=K R_{2 d o w n}\left(i \omega, k_{x},-k_{y}\right) K
$$

where

$$
K=\left(\begin{array}{cc}
G_{1} & 0 \\
0 & G_{1}
\end{array}\right)
$$

with matrix elements $e^{-L \sqrt{\omega^{2}+k_{y}^{2}+\left(k_{x}+\frac{2 \pi p}{d}\right)^{2}}}, p=-N \ldots N$, on the main diagonal of a matrix $G_{1}$. Note that in the formula (7) the matrix $R_{2 \text { down }}$ is a reflection matrix obtained from the mirror image of the second grating ( $z=L / 2$ is the location of a mirror), the mirror image of the second grating is located at $z<0$. It is sufficient then to make a change of coordinates $z \rightarrow L-z_{1}, y \rightarrow-y_{1}$ in Rayleigh decompositions for the mirror image grating to obtain the reflection matrix for the upper grating $R_{2 u p}$.

The Casimir free energy for $1 \mathrm{~d}$ gratings separated by a vacuum slit can be written as a generalization of the zero temperature result (6):

$$
\mathcal{F}=T \sum_{n=0}^{\infty} \int_{-\infty}^{\infty} \frac{d k_{y}}{2 \pi} \int_{-\pi / d}^{\pi / d} \frac{d k_{x}}{2 \pi} \ln \operatorname{det}\left(I-R_{1 \text { down }}\left(i \omega_{n}, k_{x}, k_{y}\right) R_{2 u p}\left(i \omega_{n}, k_{x}, k_{y}\right)\right),
$$

here $T$ is the temperature of the system, $\omega_{n}=2 \pi n T$ are Matsubara frequencies; $R_{1 \text { down }}, R_{2 u p}$ are matrices of reflection coefficients from the lower and the upper gratings correspondingly, the prime means that the zero Matsubara term is taken with the coefficient $1 / 2$.

\section{Diffraction from a Chern-Simons layer}

The Chern-Simons action for a plane layer at $z=0$ is

$$
S=\frac{a}{2} \int \varepsilon^{z v \rho \sigma} A_{v} F_{\rho \sigma} d t d x d y,
$$

with the current $J^{v}=a \varepsilon^{z \nu \rho \sigma} F_{\rho \sigma}$. Equations for the electromagnetic field in the presence of Chern-Simons action (10) can be written as:

$$
\partial_{\mu} F^{\mu \nu}+a \varepsilon^{z v \rho \sigma} F_{\rho \sigma} \delta(z)=0
$$


From (11) one can derive boundary conditions for a flat Chern-Simons layer at $z=0$ in the vacuum [18] :

$$
\begin{aligned}
\left.E_{z}\right|_{z=0^{+}}-\left.E_{z}\right|_{z=0^{-}} & =-\left.2 a H_{z}\right|_{z=0}, \\
\left.H_{x}\right|_{z=0^{+}}-\left.H_{x}\right|_{z=0^{-}} & =\left.2 a E_{x}\right|_{z=0}, \\
\left.H_{y}\right|_{z=0^{+}}-\left.H_{y}\right|_{z=0^{-}} & =\left.2 a E_{y}\right|_{z=0} .
\end{aligned}
$$

Consider a diffraction of an electromagnetic wave in $s$ (TE) polarization from the ChernSimons layer located at $z=0$ (the multiplier $\exp \left(i \omega t+i k_{y} y\right)$ is omitted in what follows):

$$
\begin{aligned}
& E_{x}=\exp \left(-i k_{z} z\right)+r_{s} \exp \left(i k_{z} z\right), z>0 \\
& E_{x}=t_{s} \exp \left(-i k_{z} z\right), z<0 \\
& H_{x}=r_{s \rightarrow p} \exp \left(i k_{z} z\right), z>0 \\
& H_{x}=t_{s \rightarrow p} \exp \left(-i k_{z} z\right), z<0
\end{aligned}
$$

From the condition (13) one gets

$$
r_{s \rightarrow p}-t_{s \rightarrow p}=2 a t_{s}
$$

From the condition $\left.E_{x}\right|_{z=0^{+}}=\left.E_{x}\right|_{z=0^{-}}$it is immediate to obtain

$$
1+r_{s}=t_{s}
$$

From the condition $\left.E_{y}\right|_{z=0^{+}}=\left.E_{y}\right|_{z=0^{-}}$and the Maxwell equation $E_{y}=-\frac{1}{i \omega} \partial_{z} H_{x}$ the equality

$$
r_{s \rightarrow p}=-t_{s \rightarrow p}
$$

follows. From the condition (14) and the Maxwell equation $H_{y}=\frac{1}{i \omega} \partial_{z} E_{x}$ one derives

$$
1-r_{s}-t_{s}=-2 a t_{s \rightarrow p}
$$

By solving the equations (19)-(22) one finds reflection and transmission coefficients for diffraction of TE electromagnetic wave on a Chern-Simons layer located at $z=0$ [9]:

$$
\begin{array}{ll}
r_{s}=-\frac{a^{2}}{1+a^{2}}, & t_{s}=\frac{1}{1+a^{2}}, \\
r_{s \rightarrow p}=\frac{a}{1+a^{2}}, & t_{s \rightarrow p}=-\frac{a}{1+a^{2}} .
\end{array}
$$

Consider a diffraction of an electromagnetic wave in $p(\mathrm{TM})$ polarization from the ChernSimons layer located at $z=0$ :

$$
\begin{aligned}
& H_{x}=\exp \left(-i k_{z} z\right)+r_{p} \exp \left(i k_{z} z\right), z>0 \\
& H_{x}=t_{p} \exp \left(-i k_{z} z\right), z<0 \\
& E_{x}=r_{p \rightarrow s} \exp \left(i k_{z} z\right), z>0 \\
& E_{x}=t_{p \rightarrow s} \exp \left(-i k_{z} z\right), z<0
\end{aligned}
$$

From the condition $\left.E_{x}\right|_{z=0^{+}}=\left.E_{x}\right|_{z=0^{-}}$it follows

$$
r_{p \rightarrow s}=t_{p \rightarrow s} .
$$

From the condition $\left.E_{y}\right|_{z=0^{+}}=\left.E_{y}\right|_{z=0^{-}}$and equation $E_{y}=-\frac{1}{i \omega} \partial_{z} H_{x}$ one gets

$$
1-r_{p}=t_{p}
$$


From the condition (13) it follows

$$
1+r_{p}-t_{p}=2 a r_{p \rightarrow s}
$$

From the condition (14) and Maxwell equation $H_{y}=\frac{1}{i \omega} \partial_{z} E_{x}, E_{y}=-\frac{1}{i \omega} \partial_{z} H_{x}$ it is immediate to obtain

$$
r_{p \rightarrow s}+t_{p \rightarrow s}=2 a t_{p}
$$

By solving the system of equations (28)-(31), one gets reflection and transmission coefficients for diffraction of TM electromagnetic wave on a Chern-Simons layer located at $z=0$ [9]:

$$
\begin{array}{ll}
r_{p}=\frac{a^{2}}{1+a^{2}}, & t_{p}=\frac{1}{1+a^{2}}, \\
r_{p \rightarrow s}=\frac{a}{1+a^{2}}, & t_{p \rightarrow s}=\frac{a}{1+a^{2}} .
\end{array}
$$

\section{Casimir energy of two Chern-Simons layers}

Reflection matrix $R_{1 \text { down }}=R\left(a_{1}\right)$ from Chern-Simons layer located at $z=0$ is defined by [9]:

$$
R\left(a_{1}\right)=\left(\begin{array}{cc}
r_{s} & r_{p \rightarrow s} \\
r_{s \rightarrow p} & r_{p}
\end{array}\right)=\frac{a_{1}}{1+a_{1}^{2}}\left(\begin{array}{cc}
-a_{1} & 1 \\
1 & a_{1}
\end{array}\right) .
$$

Reflection matrix from Chern-Simons layer at $z=L$ is defined after euclidean rotation by

$$
R_{2 u p}\left(-a_{2}\right)=S R\left(a_{2}\right) S
$$

where

$$
S=\left(\begin{array}{cc}
e^{-L \sqrt{\omega^{2}+k_{x}^{2}+k_{y}^{2}}} & 0 \\
0 & e^{-L \sqrt{\omega^{2}+k_{x}^{2}+k_{y}^{2}}}
\end{array}\right)
$$

is a matrix due to change of the coordinate system $x_{1}=x, y_{1}=-y, z_{1}=-z+L, L$ is a distance between Chern-Simons layers.

The Casimir energy of two Chern-Simons layers in vacuum is

$$
\begin{gathered}
E\left(a_{1},-a_{2}, l\right)=\frac{1}{2} \iiint \frac{d \omega d k_{x} d k_{y}}{(2 \pi)^{3}} \ln \operatorname{det}\left(I-R_{1 \text { down }}\left(a_{1}\right) R_{2 u p}\left(-a_{2}\right)\right)= \\
\frac{1}{4 \pi^{2}} \int_{0}^{+\infty} d r r^{2} \ln \operatorname{det}\left(I-e^{-2 L r} R\left(a_{1}\right) R\left(a_{2}\right)\right)= \\
\frac{1}{4 \pi^{2}} \int_{0}^{+\infty} d r r^{2} \ln \operatorname{det}\left(I-e^{-2 L r} Q\right),
\end{gathered}
$$

where

$$
Q=a_{1} a_{2}\left(\begin{array}{cc}
\frac{1}{\left(a_{1}-i\right)\left(a_{2}+i\right)} & 0 \\
0 & \frac{1}{\left(a_{1}+i\right)\left(a_{2}-i\right)}
\end{array}\right) .
$$

Performing the integrals one gets for the energy [9]:

$$
E\left(a_{1},-a_{2}, L\right)=-\frac{1}{16 \pi^{2} L^{3}}\left(\operatorname{Li}_{4}\left(\frac{a_{1} a_{2}}{\left(a_{1}-i\right)\left(a_{2}+i\right)}\right)+\operatorname{Li}_{4}\left(\frac{a_{1} a_{2}}{\left(a_{1}+i\right)\left(a_{2}-i\right)}\right)\right),
$$

where $\operatorname{Li}_{4}(x)=\sum_{k=1}^{+\infty} x^{k} / k^{4}=-\frac{1}{2} \int_{0}^{+\infty} d r r^{2} \ln \left(1-x e^{-r}\right)$. 
The plane with Chern-Simons term has a chosen direction, and changing the parameter $a_{2} \rightarrow-a_{2}$ in the Chern-Simons term is equivalent to rotating the Chern-Simons plane around any line passing through it by 180 degrees. Note that $E\left(a_{1}, a_{2}\right)$ is the Casimir energy of two Chern-Simons layers with equal chosen directions.

For $a_{1}=-a_{2}$ the force is attractive for every $a_{1}$.

For $a_{1}=a_{2}$ one gets the Casimir energy of two Chern-Simons layers with identically selected directions of layers in space [8]. In this case the force is repulsive at all distances $L$ for $a_{1} \in\left[0, a_{0}\right]$, where $a_{0} \approx 1.032502$, and attractive at all distances $L$ for $a_{1}>a_{0}$.

\section{Acknowledgments}

This research was performed by use of computational resources provided by Resource Center "Computer Center of SPbU" (http://cc.spbu.ru/en) through a grant of Saint Petersburg State University No.11.40.538.2017.

\section{References}

[1] H. B. G. Casimir, Proc. Kon. Ned. Akad. Wetensch. 51, 793-795 (1948).

[2] E. M. Lifshitz, ZhETF 29, 94 (1955); E. M. Lifshitz, Soviet Phys. JETP 2, 73 (1956).

[3] Yu. S. Barash and V. L. Ginzburg, Sov. Phys. Usp. 18, 305 (1975); Sov. Phys. Usp. 27, 467 (1984).

[4] G. Plunien, B. Müller and W. Greiner, Phys.Rept. 134, 87 (1986).

[5] K. A. Milton, J.Phys.A: Math. Gen. 37, R 209 (2004).

[6] R. L. Jaffe, Phys. Rev. D 72, 021301(R) (2005).

[7] V. N. Marachevsky, J.Phys.A: Math. Theor. 45, 374021 (2012).

[8] V. N. Markov and Yu. M. Pis'mak, J. Phys. A: Math. Gen. 39, 6525 (2006).

[9] V. N. Marachevsky, Theor.Math.Phys. 190(2), 315 (2017).

[10] V. N. Marachevsky and Yu. M. Pis'mak, Phys.Rev.D 81, 065005 (2010).

[11] S. Y. Buhmann, V. N. Marachevsky and S. Scheel, ArXiv: 1803.01069 .

[12] V. N. Marachevsky, ArXiv: 1802.06523.

[13] A. Lambrecht and V. N. Marachevsky, Phys.Rev.Lett. 101, 160403, 1-4 (2008).

[14] A. Lambrecht and V. N. Marachevsky, Int. J. Mod. Phys. A 24, 1789-1795 (2009).

[15] O. M. Rayleigh, Proc. Roy. Soc. A 79, 399-416 (1907).

[16] H.-C. Chiu, G. L. Klimchitskaya, V. N. Marachevsky, V. M. Mostepanenko and U. Mohideen, Phys. Rev. B 80, 121402(R) (2009); Phys. Rev. B 81, 115417 (2010).

[17] D. Maystre (ed.), Selected Papers on Diffraction Gratings (SPIE, Bellingham, 1993);

R. Petit (ed.), Electromagnetic Theory of Gratings (Springer-Verlag, Berlin, 1980);

M. C. Hutley, Diffraction Gratings (Academic Press, London, 1982); E. G. Loewen and

E. Popov, Diffraction Gratings and Applications (Marcel Dekker, New York, 1997).

[18] D. Yu. Pis'mak, Yu. M. Pis'mak and F. J. Wegner, Phys.Rev.E 92, 013204 (2015). 